Journal of Korean Powder Metallurgy Institute

Vol. 18, No. 3, 2011

DOI: $10.4150 /$ KPMI.2011.18.3.297

\title{
Comparative Analyses of Commercial Detonation Nanodiamonds
}

\author{
A. P. Puzyr, A. E. Burov ${ }^{a}$, V. S. Bondar, C. K. Rhee ${ }^{b, *}$, W. H. Rhee ${ }^{c}$, and K. C. Hwang \\ Institute of Biophysics SB RAS, Krasnoyarsk 660036, Russia \\ ${ }^{a}$ Special Design-Technology Bureau "Nauka” SB RAS, Krasnoyarsk, Russia \\ ${ }^{b}$ Korean Atomic Energy Research Institute, Daejeon 305-353, Korea \\ ${ }^{c}$ Daewha Alloytech. Co., Gyeonggi-do 445-862, Korea
}

(Received April 25, 2011; Revised May 17, 2011; Accepted June 8, 2011)

\begin{abstract}
Colloidal stability is one of crucial factors for many applications of nanodiamond. Despite recent development, nanodiamonds available on the market often exhibit a high impurity content, wide size distribution of aggregates and low resistance to sedimentation. In the current study, four commercial nanodiamond powders synthesized by detonation synthesis were surface modified and then separated with respect to the size into six fractions by centrifugation. The fractions were evaluated by zeta potential, particle size distribution and elemental composition. The results showed that the modified nanodiamonds form stable colloidal suspensions without sedimentation for a long time.
\end{abstract}

Keywords : Nanoparticles, Detonation nanodiamond, Surface modification, Colloidal stability

\section{Introduction}

Detonation nanodiamond (DND) is produced by detonation synthesis from carbon-containing explosives in an oxygen depleted atmosphere [1]. The material is receiving much attention due to its remarkable mechanical, electrical and thermal properties, facile surface tailoring, narrow size distribution of primary particles and high biocompatibility [2]. Nanodiamonds find wide applications in composite coatings for metals, drug delivery vehicles, diagnostic probes, micro-electro-mechanical devices, additives to polymers and lubricants etc [2,3].

Despite recent development, DNDs available on the market often require additional treatment as the impurity content is usually high, size distribution of aggregates is wide, and resistance to sedimentation is low. While being stable in aqueous solution, upon drying DND particles coagulate and lose colloidal stability. This makes impossible their subsequent resuspension or fractionating. It stipulates storage and transportation of DND only in solutions at relatively low concentrations ( $\max 10 \mathrm{wt} \%$ ).

Colloidal stability is one of crucial factors for many applications of DND including but not limited to drug delivery systems, entesorbents, different types of injections, extraction and purification of proteins, indicator systems, lubricant additives, polishing compositions and CVD precursors. Moreover, there is often the need for a certain particle size of DND [2-5]. For this purpose the centrifugal fractionation is often applied, which requires stable colloids.

Colloidal stability of commercial nanodiamonds can be achieved through the size reduction of aggregates and/or increase of repulsive inter-particle forces. Several approaches were developed so far to improve the sedimentation resistance of DND in solutions including the use of surfactants, purification by ion exchange and heat treatment [6-9]. However, most of them are limited to certain solvents (usually non-aqueous), or require sophisticated equipment, high energy consumption, and hence, are

*Corresponding Author : [Tel : +82-42-868-8551; E-mail : ckrhee@kaeri.re.kr] 
expensive. Moreover, subsequent drying may lead to the loss of ability of DND to form a stable suspension [8].

In the current study, four commercial DND powders were chemically treated to decrease aggregate size and modify the surface of nanoparticles. Analyses of XRD, SEM and TEM were carried out to investigate the colloidal stability, fractional and elemental compositions of the modified nanodiamonds.

\section{Experimental}

Unfractioned detonation nanodiamond powders produced in Russia (two samples) and China (two samples) are denoted as $\mathrm{Ru} 1, \mathrm{Ru} 2, \mathrm{Ch} 1, \mathrm{Ch} 2$ in the following text, respectively. The incombustible impurity contents in $\mathrm{Ch} 1$ and $\mathrm{Ch} 2$ powders provided by the producers were 1.19 and $0.52 \%$, respectively. The elemental composition of Ru1 and Ru2 powders was determined experimentally.

All acquired DNDs are heterogeneous, polydisperse systems and exhibit loosely and tightly bound aggregates up to $5 \mu \mathrm{m}$ in diameter (Fig. 1). The former are formed by the adsorption between the nanoparticles, while the latter are caused by chemical bindings.

The DND powders were treated using a method describe previously $[10,11]$. Briefly, the modifica-

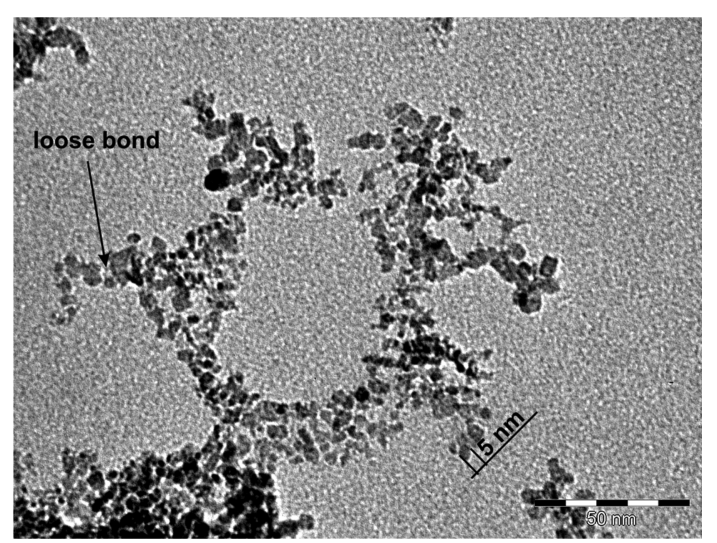

Fig. 1. TEM image of the nanodiamond(ND) produced by detonation synthesis in Russia (Ru1). tion and simultaneous fractionating started with preparation of the initial hydrosol that included deionized water (Milli-Q system, $18 \mathrm{M} \Omega \mathrm{cm}$ ), a DND powder at concentrations of $1-3 \mathrm{wt} \%$ and a modifying reagent (10 mg per $1 \mathrm{~g}$ of DND). Ethylenediaminetetraacetic acid (EDTA) and sodium chloride $(\mathrm{NaCl})$ were used as the modifiers. Ultrasonic pretreatment of the initial hydrosol was excluded for two reasons: the process is time and cost consuming; sonication inevitably results in wear debris from the sonotrode tip, which is difficult to remove.

The suspension was held at room temperature for 1 hour. During this time DND particles fully precipitated and reaction products between the modifier and surface substances of DND were released into the supernatant. The precipitate was treated with water, intensively agitated with a stirrer and then centrifuged. Centrifugal fractionation was performed using a Beckman Coulter Avanti J-E centrifuge at $10{ }^{\circ} \mathrm{C}$. Six fractions were obtained from each sample. The fractionating scheme is presented in Fig. 2. The final centrifuging (F2 fraction) was done for additional

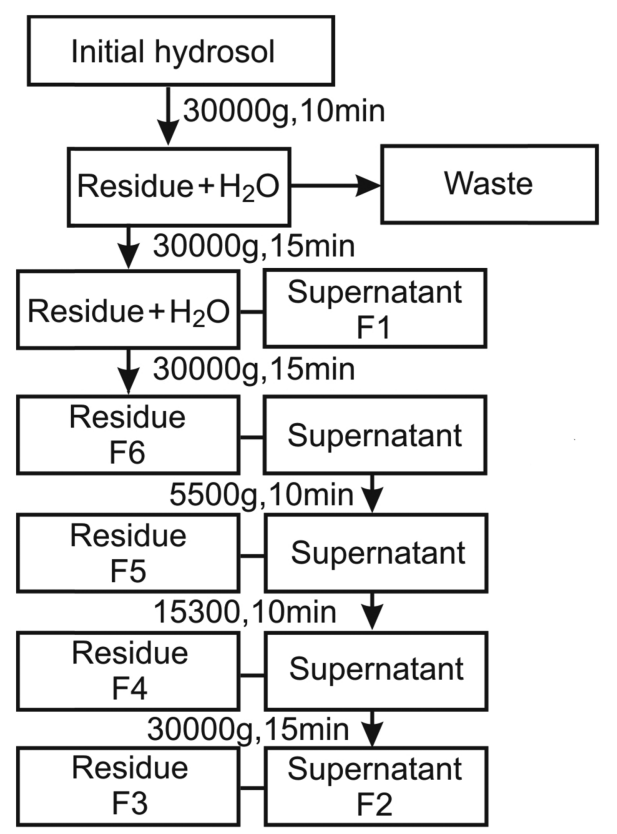

Fig. 2. Centrifugation scheme for purification and fractioning of nanodiamond. 
extraction of small particles that were "seized" by the larger aggregates during segregation. The obtained hydrosols were dried at $100^{\circ} \mathrm{C}$ for $24 \mathrm{~h}$. Subsequently, the powders were weighed to determine the mass yield of each fraction.

The DND size distribution within the fractions was determined at $22^{\circ} \mathrm{C}$ by the dynamic light scattering technique with the Beckman Coulter \#5 analyzer. Zeta potential measurements were performed on Particle Size Analyzer 90PLus (Brookhaven Instruments Corporation). The reported results were averaged over 5 measurements.

X-ray diffraction (XRD), scanning electron microscopy (SEM) and transmission electron microscopy (TEM) were used to study the composition and structure of DND fractions.

\section{Results and Discussion}

The stability of hydrosols prepared from all asreceived powders was poor. Fig. 3 presents $2 \mathrm{wt} \%$ suspension of the starting Rul powder just after dispersion (a) and one day later (b). Most of nanoparticles precipitated making centrifugation fractionation impossible. We suppose that some of the impurities remained after extraction of DND from the detonation soot promote their aggregation and, thus, deteriorate the stability of nanodiamond suspensions.

The modification imparted DNDs with a high colloidal stability and no detectable precipitation was found after at least three months. The stability was
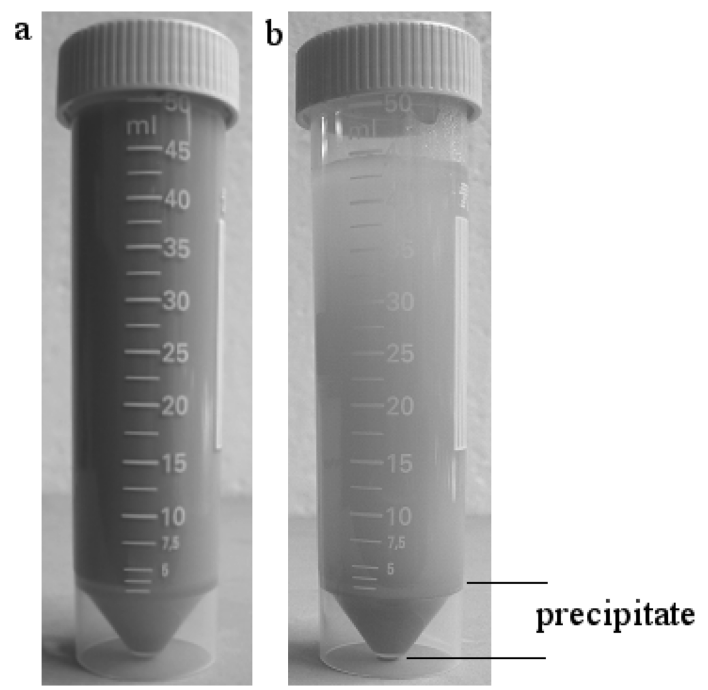

Fig. 3. Hydrosol with $2 \mathrm{wt} \%$ nanodiamond (Ru1 sample) : $a$, initial and $b$, after $24 \mathrm{~h}$.

confirmed by high absolute values of zeta potential measured on $0.1 \mathrm{wt} \%$ suspensions (Table 1). The origin of the negative zeta potential of DND is discussed elsewhere [5]. After repeated drying and resuspending in water, stable hydrosols were obtained.

The particle size distribution within the fractions are shown in Table 1. These data demonstrate that centrifugation can be used to separate the initial powder in fractions of a certain average particle size. However, a relatively high degree of polydispersity evidences that the procedure is not very selective due mainly to extremely irregular shape of DND aggregates (Fig. 1). To narrow the size distribution an additional fractionation with appropriate centrifu-

Table 1. Properties of fractions with different sizes of modified nanodiamonds

\begin{tabular}{|c|c|c|c|c|c|}
\hline Fraction & $\begin{array}{c}\text { Mean diameter*, } \\
\mathrm{nm}\end{array}$ & $\begin{array}{l}\text { Polydis- } \\
\text { persity* }\end{array}$ & $\begin{array}{c}\text { Zeta potential*, } \\
\mathrm{mV}\end{array}$ & $\begin{array}{c}\text { Powder } \\
\text { appearance }\end{array}$ & Hydrosol appearance \\
\hline $\mathrm{F} 1$ & 70 & 0.21 & -51.4 & Black & Dark brown or black, transparent \\
\hline $\mathrm{F} 2$ & 70 & 0.21 & -50.0 & Black & Dark brown or black, transparent \\
\hline F3 & 145 & 0.17 & -49.2 & Dark grey & Dark grey or light brown, turbid \\
\hline $\mathrm{F} 4$ & 175 & 0.16 & -51.1 & Light grey & Grey, turbid \\
\hline F5 & 250 & 0.18 & -33.9 & Brown & Light grey or milky, turbid \\
\hline F6 & $>400$ & 0.23 & -24.6 & Milk white or beige & Milky, turbid \\
\hline
\end{tabular}

*average value obtained from measurements 
Table 2. Percentage of impurities in the initial and modified powders

\begin{tabular}{lcccc}
\hline \hline \multirow{2}{*}{ Element } & \multicolumn{2}{c}{ Ru1 } & \multicolumn{2}{c}{ Ru2 } \\
\cline { 2 - 5 } $\mathrm{Mg}$ & Initial & Modified & Initial & Modified \\
$\mathrm{Al}$ & - & - & 0.21 & 0.061 \\
$\mathrm{Si}$ & 0.03 & 0.03 & 0.07 & 0.07 \\
$\mathrm{P}$ & 0.03 & 0.02 & 0.22 & 0.212 \\
$\mathrm{~S}$ & - & 0.03 & 0.05 & 0.05 \\
$\mathrm{~K}$ & - & - & 0.07 & 0.039 \\
$\mathrm{Cl}$ & - & - & 0.01 & 0.01 \\
$\mathrm{Ca}$ & 0.25 & 0.012 & 0.77 & 0.102 \\
$\mathrm{Cr}$ & - & - & 0.03 & 0.022 \\
$\mathrm{Fe}$ & 0.67 & 0.481 & 0.6 & 0.503 \\
$\mathrm{Ni}$ & 0.02 & 0.02 & 0.01 & 0.01 \\
$\mathrm{Cu}$ & 0.12 & 0.096 & 0.04 & 0.029 \\
$\mathrm{Zn}$ & 0.01 & - & - & - \\
\hline
\end{tabular}

gation conditions is required.

As result of the modification, the level of inorganic contamination was reduced (Table 2). The improved purification can be attributed to the ability of the modifiers to sequester metal ions in aqueous solutions.

All tested materials exhibit a feature that is intrinsic for DND: the color of a powder depends on the average size of particles. It changes from a deep black for the smallest fraction through grey for the middle sized fraction to milk white or beige for the biggest one (Table 1). Traditionally $[5,10]$, fractions F1-F3 are called "black" or "dark" and fractions F4F6 are called "grey". In terms of serviceableness, the smaller the aggregates the more applications they can be used for, as percentage of non-diamond carbon and impurities is relatively low, and composition of surface groups is more uniform [5]. That is especially important for biomedical use of DND. However, for some applications, such as lubricant additives or polishing agents, 200-400 nm particles can also be applied.

The fraction analysis shows that all DND powders are polydispersed systems containing aggregates of

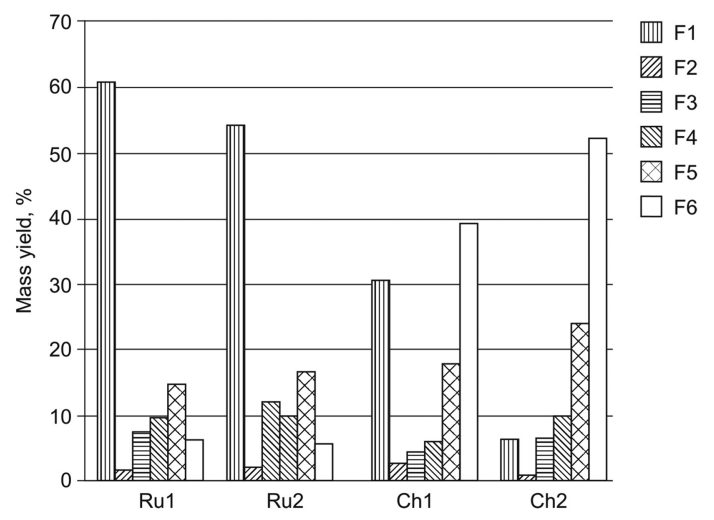

Fig. 4. Fraction compositions of nanodiamond powders.

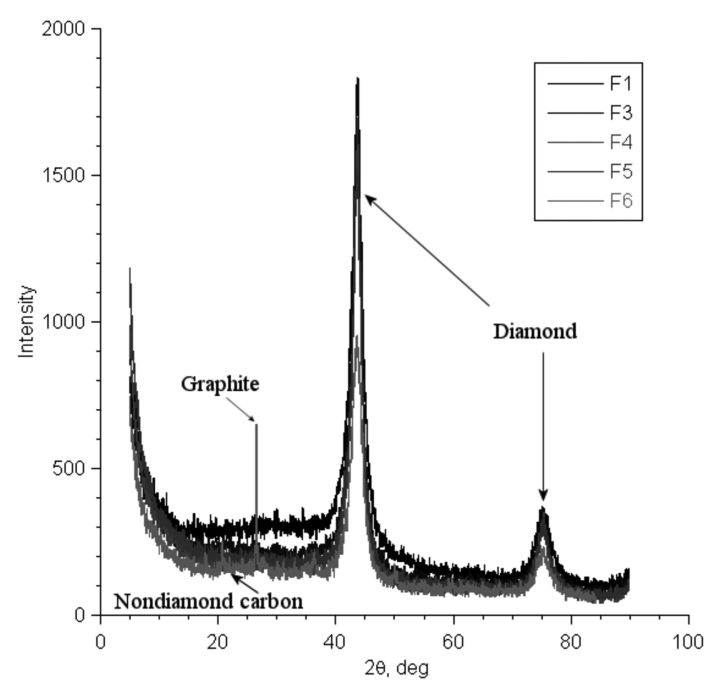

Fig. 5. X-ray diffraction patterns of fractions obtained from Ru2 samples.

different sizes. However, the yield of individual fractions varies significantly between DND powders (Fig. 4). The smallest F1 and F2 fractions comprise up to about $60 \%$ of Ru1 and Ru2 samples. On the contrary, the Ch1 and $\mathrm{Ch} 2$ samples contain $40 \%$ and $52 \%$ of the largest F6 fraction, respectively. This can be explained by different methods of the synthesis and soot purification used by the producers.

Besides the difference in size, the fractions significantly differ in composition. As it is seen on XRD patterns (Fig. 5) obtained on Ru2 samples, fraction F1 comprises mostly of the diamond carbon (reflections from the (111) and (220) planes at $2 \theta=44^{\circ}$ and 


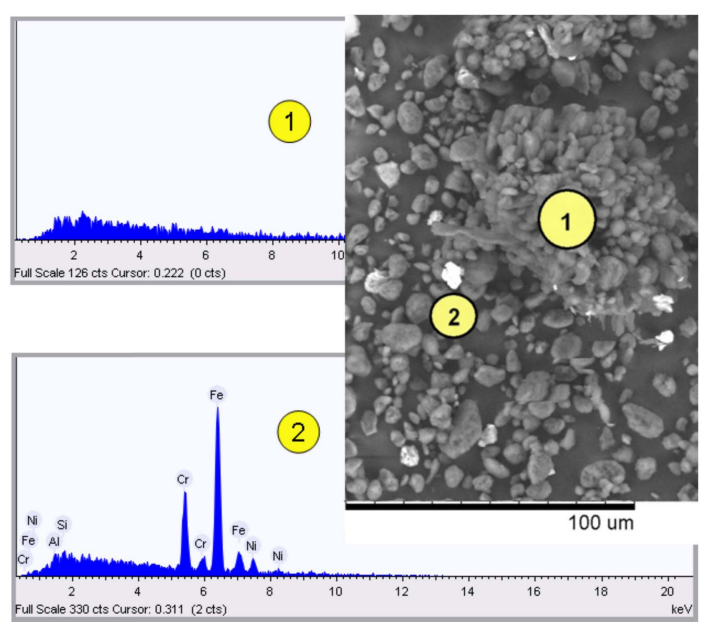

Fig. 6. SEM images and elemental compositions of the F6 fraction of the $\mathrm{Ch} 2$ powder.

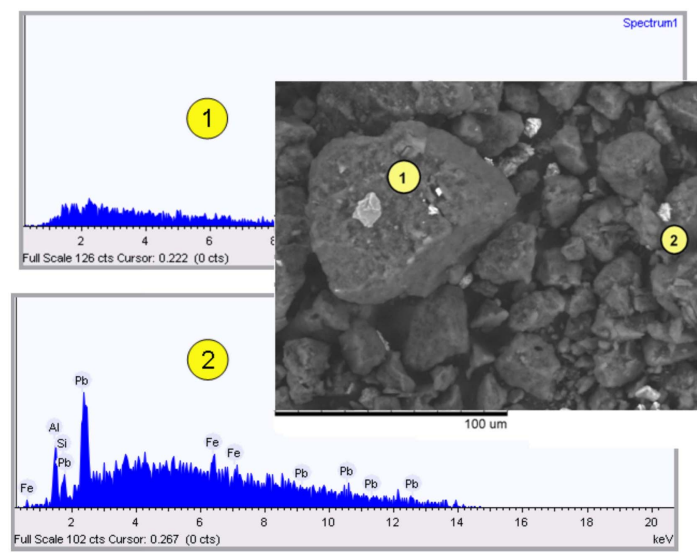

Fig. 7. SEM images and elemental compositions of the F6 fraction of the Ru2 powder.

$76^{\circ}$ respectively,). It includes little of nondiamond carbon such as graphite nanocrystals, carbon onions and amorphous carbon which presence is evident from reflection at $2 \theta=20^{\circ}-30^{\circ}$. The content of nondiamond carbon gradually increases in larger fractions. Interesting is the fact that F1 powder is black and, for example, F4 is grey. Therefore, the nondiamond carbon content does not correlate with the color of a powder and its hydrosol.

All tested powders include relatively small amount of micron-sized particles contained within the F6 fraction. They in quantities incorporate nondiamond carbon, metals and other incombustible impurities, which originate mostly from a detonator and the walls of the detonation chamber (Fig. 6, 7). As the key requirement for the chemical composition of modern DND is a minimum of incombustible impurities, such large particles must be removed. Since the impurities are not exposed for acid of heat treatment this can be achieved only by fractionating. Figures 6 and 7 show SEM images and elemental compositions obtained on the F6 samples.

\section{Conclusions}

Four DND powders from different vendors were evaluated in terms of hydrosol stability, fraction and elemental composition. In all cases, the stability of hydrosols prepared from the as-received polydispersed DND powders was low.

The initial DNDs were modified to acquire sedimentation stability and were separated by centrifugation in six fractions varied by the average size and mass yield. The modified DNDs are characterized by a high colloidal stability and form stable hydrosols even when water is simply added without ultrasonic dispersion. After repeated drying and re-suspending in water, a stable hydrosol can be obtained. The modified DND can be used "as is" or be a basic material for further treatment towards certain applications.

The study revealed that properties of the powders such as the fraction composition and impurity content are significantly different. This confirms a wellknown fact that a universal detonation nanodiamond does not exist and commercial products are specific to the synthesis and post-synthesis methods adapted by the producers.

It should be mentioned that the modification did not always leads to desired results and there were examples of commercial DND, which did not exhibit colloidal stability after the modification. The authors believe that the content of impurities (especially metallic ones) is responsible for such behavior. 


\section{Acknowledgment}

The research was financially supported by KoreaRussia Technology Development Cooperation Program (Commercialization Short Period R\&D) in the area of components and materials from Ministry of Knowledge Economy (MKE) of Republic of Korea.

\section{References}

[1] V. V. Danilenko: Phys. Solid State, 46 (2004) 595.

[2] A. M. Schrand, S. A. C. Hens and O. A. Shenderova: Critical Reviews in Solid State and Mat. Sci., 34 (2009) 18.

[3] O. A. Shenderova and D. M. Gruen (Eds.): William Andrew Publishing, (2006).

[4] M.Yu. Koroleva, D. V. Berdnikova, B. V. Spitsyn and
E. V. Yurtov: Theor. Found. of Chem. Eng., 43 (2009) 478.

[5] N. Gibson, O. Shenderova, T. J. M. Luo, S. Moseenkov, V. Bondar, A. Puzyr, K. Purtov, Z. Fitzgerald and D. Brenner: Diamond Relat. Mater., 18 (2009) 620.

[6] M. Ozawa,M. Inaguma, M. Takahashi, F. Kataoka, A. Krüger and E. Osawa: Adv. Mater., 19 (2007) 1201.

[7] I. Petrov, O. Shenderova, V. Grishko, V. Grichko, T. Tyler, G. Cunningham and G. Mcguire: Diamond Relat. Mater., 16 (2007) 2098.

[8] E. Õsawa: Pure Appl. Chem., 80 (2008) 1365.

[9] Y. Liang, T. Meinhardt, G. Jarre, M. Ozawa, P. Vrdoljak, A. Schöll, F. Reinert and A. Krueger: J. of Colloid and Interface Science, 354 (2011) 23.

[10] V. S. Bondar and A. P. Puzyr: Phys. Solid State, 46 (2004) 716.

[11] A. P. Puzyr and V. S. Bondar: Russia, RU Patent 2252192 (2003). 